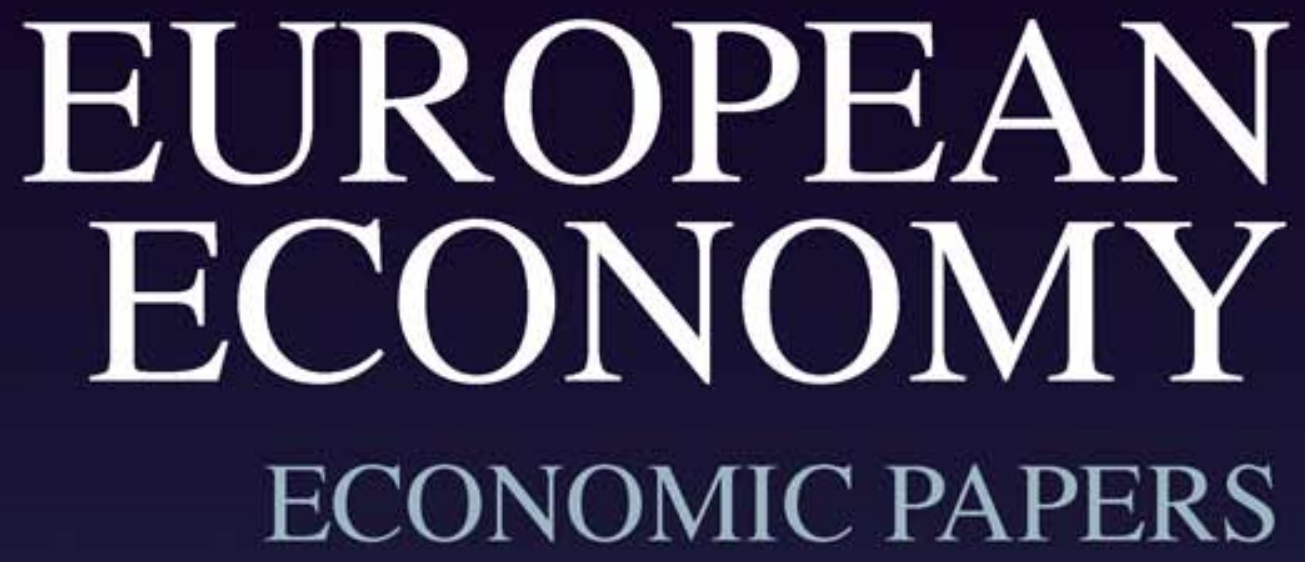

Number 278 - May 2007

Robust Monetary Policy with the Cost Channel

by Peter Tillmann (University of Bonn) 
Economic Papers are written by the Staff of the Directorate-General for Economic and Financial Affairs, or by experts working in association with them. The "Papers" are intended to increase awareness of the technical work being done by the staff and to seek comments and suggestions for further analyses. Views expressed represent exclusively the positions of the author and do not necessarily correspond to those of the European Commission. Comments and enquiries should be addressed to the:

European Commission

Directorate-General for Economic and Financial Affairs

Publications

BU1 -

B - 1049 Brussels, Belgium

ISBN 978-92-79-04631-5

KC-AI-07-278-EN-N

CEuropean Communities, 2007 


\title{
Robust Monetary Policy with the Cost Channel
}

\author{
Peter Tillmann ${ }^{1}$ \\ University of Bonn
}

April 2007

\begin{abstract}
Recent research argues that model uncertainty leads the central bank to adjust interest rates stronger to exogenous disturbances than under certainty. This paper investigates whether the introduction of a cost channel of monetary transmission, whose presence is empirically supported, changes the impact of model uncertainty on interest rate setting. The basic model is simple enough to facilitate an analytical closed form solution. We find that the presence of the cost channel dampens the effect of model uncertainty on interest rate setting and can even offset the activist policy stance. In this case, the conventional result is reversed and uncertainty induces an attenuated interest rate policy. A richer dynamic model corroborates these findings.
\end{abstract}

Keywords: model uncertainty, robust control, cost channel, optimal monetary policy, Brainard conservatism

JEL classification: E31, E32

\footnotetext{
${ }^{1}$ University of Bonn, Institute for International Economics, Lennéstr. 37, D-53113 Bonn, E-mail: tillmann@iiw.uni-bonn.de

The paper was partly written while I was visiting fellow at the European Commission (DG ECFIN) in October 2006. I thank seminar participants at DG ECFIN, especially Werner Röger, and the fifth meeting of the DFG network "Quantitative Macroeconomics" in Berlin for insightful comments. All remaining errors are mine.
} 


\section{Introduction}

It is now generally acknowledged that central banks face uncertainty about the true structure of the model that best describes the economy. ${ }^{2}$ Policymakers aim at setting interest rates optimally given a particular reference model but, at the same time, admit that they cannot be completely certain about the true model specification. As a result, central banks want to formulate robust policies that are to some extent immune with respect to model disturbances. In particular, one strand of the literature on monetary policymaking under uncertainty proposes to formulate a policy that performs reasonably well even if the worst possible misspecification realizes. In other words, the policymaker is unable to formulate a probability distribution over a range of plausible models. Instead, he sets interest rates so as to minimize the maximum harm to the economy. Such a policy concept is known as a robust control approach to policymaking and was pioneered by Hansen and Sargent (2005).

The robust control approach sheds new light on the classical result of Brainard (1967). In a series of papers, Giannoni (2002, 2005) and Onatski and Stock (2002), among others, analyze whether the Brainard result carries over to robust policy in a New Keynesian model of monetary policy. Brainard argued that multiplicative parameter uncertainty should lead to an attenuated adjustment of the policy instrument. Blinder (1997, p. 11) refers to this result as the "Brainard conservatism principle". ${ }^{3}$ Recently, this principle has been challenged as the

\footnotetext{
${ }^{2}$ For a survey of optimal monetary policy under various dimensions of uncertainty see Walsh (2004).

${ }^{3}$ Blinder (1997, p. 12) further notes that "I wish more academics would train their highpowered tools on this question, for I can tell you that, as a Federal Reserve governor, I always viewed the Brainard conservatism principle as extremely wise." Wieland (2000) presents one of the first studies of optimal monetary policy under parameter uncertainty using Bayesian learning. Sack (2000) studies the interest rate setting behavior of the Federal Reserve and supports the Brainard principle empirically. Söderström (2002) finds that uncertainty about the persistence properties of inflation gives rise to more aggressive policy, while uncertainty about other parameters might dampen the policy response.
} 
literature moved to discuss robust policy under general model uncertainty. ${ }^{4}$ Giannoni $(2002,2005)$ and Onatski and Stock (2002) show that model uncertainty does no longer justify a cautious monetary policy response since the policymaker fears inflation to be higher than under certainty and, consequently, adjusts the policy instrument more aggressively. Leitemo and Söderström (2005) recently provide a tractable framework to analyze robust monetary policy within a simple New Keynesian macro model. The appealing feature of their contribution is its tractability that allows the researcher to solve the model analytically. They show that uncertainty leads to a more vigorous response to supply and demand shocks.

In this paper, we extend the model of Leitemo and Söderström to account for the cost channel of monetary transmission while preserving the paper's analytical tractability. Recent empirical research by Barth and Ramey (2001) and others draws attention to the cost channel transmission of monetary impulses to the economy, which describes a supply-side effect of monetary policy that augments the conventional demand-side channel. To the extend that firms must pay the factors of production before they receive revenues from selling their products, they rely on borrowing from financial intermediaries. Monetary policy therefore impacts on the cost side of the economy. Higher interest rates translate into higher costs of working capital and induce a rise in inflation. Recently, Ravenna and Walsh (2006) and Chowdhury, Hoffmann, and Schabert (2006) integrate a cost channel in an otherwise standard New-Keynesian model of the business cycle and show that the presence of a cost channel is tantamount to a direct effect of interest rates on the inflation rate within a forward-looking Phillips curve.

Here we introduce the cost channel of monetary transmission into a standard New Keynesian model and solve for optimal monetary policy that is robust to model misspecifications. We find that the introduction of the cost channel has

\footnotetext{
${ }^{4}$ The results of Tetlow and von zur Mühlen (2001) show that the effect of uncertainty on interest rate setting might be less clear-cut than the distinction between multiplicative parameter uncertainty and general model uncertainty suggests. In fact, Žaković, Wieland, and Rustem (2005) derive a min-max optimal policy under parameter and shock uncertainty that tends to obey the Brainard principle.
} 
important consequences for the design of monetary policy under model uncertainty. While model uncertainty, in general, leads the central bank to pursue a more aggressive policy and adjust interest stronger when facing shocks, the presence of a cost channel tends to attenuate interest rate setting behavior. We find that these two effects mutually neutralize at very plausible parameter constellations. Hence, at this point uncertainty does not matter for interest rate setting. Moreover, we find that the response to demand shocks becomes attenuated at realistic realizations of the cost channel. Hence, this paper provides one example in which the seminal Brainard result carries over to a min-max approach to optimal monetary policy in a sticky-price framework.

Central to Hansen and Sargent's robust control approach is the distinction between the policymaker's reference model and the approximating model. The reference model provides the most likely description of the economy. In the absence of model misspecifications, this model generates the conventional rational expectations solution. Under robust control, however, the policymaker believes the model to be misspecified to a certain degree. He formulates a policy rule which is robust to these model distortions and shields the economy from the worst possible misspecification. This paper introduces a cost channel into the reference model and derives a robust optimal policy. The approximating model characterizes the behavior of inflation and output if the policymaker follows the robust policy rule but the reference model turns out to be undistorted. We assess how the presence of a cost channel affects interest rate setting behavior under the robust optimal policy and evaluate the impact of uncertainty on the resulting inflation and output dynamics.

This paper is organized as follows. Section two characterizes the simple model economy while section three derives optimal monetary policy which is robust to model uncertainty. Section four calibrates the model and analyses the effect of model uncertainty on interest rate setting behavior. In section five, several simplifying restrictions are relaxed. It is shown that the basic results are supported by a richer dynamic model. Section six finally concludes. 


\section{Optimal monetary policy with a cost channel of monetary transmission}

Monetary policy shocks are usually thought of as affecting the economy through their effect on aggregate demand. In a widely cited study, Barth and Ramey (2001) provide aggregate and industry-level evidence for the conjecture that monetary policy impulses also have important supply-side effects that accompany the impact on the demand-side of the economy. This cost channel can be motivated as follows. Assume that firms have to pay their factors of production before they receive revenues from selling their products and need to borrow working capital from financial intermediaries. Thus, a rise in the short-term interest rate directly translates into higher costs of working capital. A monetary contraction affects the cost side of the economy and leads to a decline in output through an adverse supply-side effect. ${ }^{5}$

We adopt a standard forward-looking monetary model and draw on recent work of Christiano, Eichenbaum, and Evans (2005), Ravenna and Walsh (2006), and others who introduce the cost of working capital into a general equilibrium model. ${ }^{6}$ The forward-looking Phillips curve (1) and the IS curve (2) represent log-linearised equilibrium conditions of a simple sticky-price general equilibrium model

$$
\begin{aligned}
& \pi_{t}=\beta E_{t} \pi_{t+1}+\kappa\left((\sigma+\eta) x_{t}+\psi i_{t}\right)+e_{t} \\
& x_{t}=E_{t} x_{t+1}-\sigma^{-1}\left(i_{t}-E_{t} \pi_{t+1}\right)+u_{t}
\end{aligned}
$$

where $\pi_{t}$ is the inflation rate, $x_{t}$ the output gap, $i_{t}$ the risk-free nominal interest rate controlled by the central bank, and $E_{t}$ is the expectations operator. All

\footnotetext{
${ }^{5}$ Recent empirical evidence stronly supports the existence of a cost channel, see Ravenna and Walsh (2006) and Chowdhury, Hoffmann, and Schabert (2006). These papers use a GMM approach to estimate reduced-form New Keynesian Phillips curves, in which the measure of real marginal cost is supplemented by the nominal interest rate.

${ }^{6}$ See, among others, Clarida, Galí, and Gertler (1999) and Woodford (2003) for a deeper analysis and the complete derivation of this family of models based on optimizing households and firms under monopolistic competition and nominal rigidities.
} 
variables are expressed in percentage deviations from their respective steady state values. The discount factor is denoted by $\beta<1, \sigma$ is the coefficient of relative risk aversion, $\eta$ is the elasticity of labor supply, and $\kappa$, the slope coefficient of the Phillips curve, depends negatively on the degree of price stickiness. The cost-push shock $e_{t}$ and the demand shock $u_{t}$ are described by $e_{t} \sim \mathcal{N}(0,1)$ and $u_{t} \sim \mathcal{N}(0,1)$. The coefficient $\psi$ describes the direct impact of interest rates on inflation and, thus, the strength of the cost channel. In the presence of a cost channel of monetary transmission, changes in interest rates directly propagate into inflation dynamics.

Monetary policy is assumed to set interest rates in order to minimize the welfare loss due to sticky-prices which is described in terms of inflation volatility and output gap volatility weighted by the parameter $\lambda>0$

$$
\min _{i_{t}} \frac{1}{2} E_{0} \sum_{t=0}^{\infty} \beta^{t}\left[\pi_{t}^{2}+\lambda x_{t}^{2}\right]
$$

Minimizing (3) subject to the model in (1) and (2) gives a set of first-order conditions, from which the optimal policy response to shocks can be computed. ${ }^{7}$

\section{Optimal robust policy}

The central banker considers the model presented in the previous section as the reference model, which represents the most likely description of the economic structure. However, the policymaker knows that this model could be subject to a wide range of distortions. The task is to reformulate the central bank's optimization problem such that the resulting policy rule performs well even if the model deviates from the reference model. A policy that is optimal in the reference model but does not take account of possible misspecifications can turn out to be disastrous if the misspecifications realize. Under robust control, in contrast, the resulting policy rule performs sufficiently well even if the underlying economic structure does not coincide with the policymaker's reference model.

\footnotetext{
${ }^{7}$ Giordani and Söderlind (2004) provide an overview of monetary policy applications and offer a set of solution algorithms.
} 
We transform the minimization problem into a min-max problem. The central bank wants to minimize the maximum welfare loss due to model misspecifications by specifying an appropriate policy. To illustrate the problem, we introduce a fictitious second rational agent, the malevolent or evil agent, whose only goal is to maximize the central bank's loss. The evil agent chooses a model from the available set of alternative models and the central bank chooses its policy optimally. Hence, the equilibrium is the outcome of a two-person game. Note that the evil agent is a convenient metaphor for the planner's cautionary behavior. Therefore, the evil agent shares the same reference model that the central bank entertains and optimizes the same objective function. The only difference is that the evil agent wants to maximize rather than minimize the resulting loss.

The set of potential misspecifications, the control vector of the evil agent, takes the form of error terms. However, these shocks are not mere additional exogenous random innovations. Let $\mathbf{v}_{t}=\left[v_{t}^{\pi}, v_{t}^{x}\right]^{\prime}$ denote the evil agent's $(2 \times 1)$ control vector. The only constraint imposed upon the fictitious evil agent is his budget constraint requiring

$$
E_{t} \sum_{\tau=0}^{\infty} \beta^{\tau}\left[\left(v_{t+\tau}^{\pi}\right)^{2}+\left(v_{t+\tau}^{x}\right)^{2}\right] \leq \omega
$$

Hence, the parameter $\omega$ measures the amount of misspecification the evil agent has available. The model thus becomes

$$
\begin{aligned}
& \pi_{t}=\beta E_{t} \pi_{t+1}+\kappa(\sigma+\eta) x_{t}+\kappa \psi i_{t}+\left[e_{t}+v_{t}^{\pi}\right] \\
& x_{t}=E_{t} x_{t+1}-\sigma^{-1}\left(i_{t}-E_{t} \pi_{t+1}\right)+\left[u_{t}+v_{t}^{x}\right]
\end{aligned}
$$

and

$$
\min _{i_{t}} \max _{\mathbf{v}_{t}} E_{0} \sum_{t=0}^{\infty} \beta^{t}\left[\pi_{t}^{2}+\lambda x_{t}^{2}\right]
$$

Note that the control variables $\mathbf{v}_{t}$ of the evil agent are masked by the shock processes $e_{t}$ and $u_{t}$. The standard rational expectations solution for optimal monetary policy corresponds to $\omega=0$, such that the evil agent's budget is empty. 
The equilibrium dynamics of the model are found by combining this solution with the reference model. If the full amount of possible misspecifications realizes, we refer to the resulting model as the worst case model. If, on the other hand, the reference model turns out to be undistorted, we refer to the resulting model as the approximating model. A central bank concerned with robustness designs policy based on the fully distorted model. Once policy is formulated, however, the central bank acts as if there were no longer any model uncertainty.

\subsection{The policy problem}

The Lagrangian of the policy problem can be written as follows

$$
\begin{aligned}
\mathcal{L}=\pi_{t}^{2}+\lambda & x_{t}^{2}-\theta\left[\left(v_{t}^{\pi}\right)^{2}+\left(v_{t}^{x}\right)^{2}\right] \\
& -\mu_{t}^{\pi}\left(\pi_{t}-\beta E_{t} \pi_{t+1}-\kappa(\sigma+\eta) x_{t}-\kappa \psi i_{t}-e_{t}-v_{t}^{\pi}\right) \\
& -\mu_{t}^{x}\left(x_{t}-E_{t} x_{t+1}+\sigma^{-1} i_{t}-u_{t}-v_{t}^{x}\right)
\end{aligned}
$$

where $\mu_{t}^{\pi}$ and $\mu_{t}^{x}$ denote the Lagrange multipliers associated to the inflation adjustment equation and the consumption Euler equation, respectively. The Lagrange parameter $\theta$ is inversely related to $\omega$. Hence, the rational expectations case corresponds to $\theta \rightarrow \infty .^{8}$ In the following, we will loosely refer to $\theta$ as the degree of robustness or the degree of uncertainty, respectively. A lower $\theta$ means that the central bank designs a policy which is appropriate for a wider set of possible misspecifications. Therefore, a lower $\theta$ is equivalent to a higher degree of robustness. ${ }^{9}$ The central bank plays a Nash game against the evil agent, who wants to maximize the welfare loss. Due to the fact that the first order conditions for a maximum and a minimum are identical, optimization under

\footnotetext{
${ }^{8}$ In this case, the evil agent maximizes the welfare loss by choosing $\mathbf{v}_{t}=0$.

${ }^{9}$ We follow Leitemo and Söderström (2005) and allow, for reasons of tractability, the evil agent only to respond to the same variables as the policymaker, i.e. the cost shock and the demand shock. Hansen and Sargent (2005), in contrast, allow the evil agent to respond also to lagged state variables. The richer dynamic model presented below relaxes this assumption.
} 
discretion results in the following set of first-order conditions

$$
\begin{aligned}
& \frac{\partial \mathcal{L}}{\partial x_{t}}=0 \Leftrightarrow \lambda x_{t}+\kappa(\sigma+\eta) \mu_{t}^{\pi}-\mu_{t}^{x}=0 \\
& \frac{\partial \mathcal{L}}{\partial \pi_{t}}=0 \Leftrightarrow \pi_{t}-\mu_{t}^{\pi}=0 \\
& \frac{\partial \mathcal{L}}{\partial i_{t}}=0 \Leftrightarrow \kappa \psi \mu_{t}^{\pi}-\mu_{t}^{x} \sigma^{-1}=0 \\
& \frac{\partial \mathcal{L}}{\partial v_{t}^{x}}=0 \Leftrightarrow-\theta v_{t}^{x}+\mu_{t}^{x}=0 \\
& \frac{\partial \mathcal{L}}{\partial v_{t}^{\pi}}=0 \Leftrightarrow-\theta v_{t}^{\pi}+\mu_{t}^{\pi}=0
\end{aligned}
$$

which can be shown to imply

$$
\begin{aligned}
x_{t} & =-\frac{\kappa}{\lambda}(\sigma(1-\psi)+\eta) \pi_{t} \\
v_{t}^{\pi} & =\frac{1}{\theta} \pi_{t} \\
v_{t}^{x} & =\psi \sigma \kappa \frac{1}{\theta} \pi_{t}
\end{aligned}
$$

Condition (14) collapses to the standard trade-off characterizing optimal discretionary monetary policy once we shut-off the cost channel, i.e. if $\psi=0$. Thus, as Ravenna and Walsh (2006) note, with $\psi>0$, optimal policy will result in greater inflation variability for a given level of output gap variability since, due to the effect of interest rates on inflation, stabilizing inflation is more costly. In other words, for a given level of inflation volatility, output variability will be lower. Note that this optimal trade-off is not affected by uncertainty.

Conditions (15) and (16) describe the evil agent's choice of model perturbations. The higher the degree of uncertainty, the larger the distortions $v_{t}^{\pi}$ and $v_{t}^{x}$. Moreover, without the cost channel, the distortion in the IS curve equals zero. Hence, in the presence of the cost channel, uncertainty about output dynamics specified in the IS curve matters for optimal policy. Without the cost channel, on the other hand, optimal policy is not affected by uncertainty about the demand side. We state a first finding:

Result 1: The more important the cost channel becomes, the larger the perceived amount of misspecification in the IS curve the central 
bank rationally fears. Both model perturbations increase with the variances of the shock processes.

If $\psi=0$, the evil agent will not disturb the IS curve and the results are identical to those obtained by Leitemo and Söderström (2005). Since the cost channel constitutes a direct link between the IS and the Phillips curve, the evil agent can do more harm if he increases the amount of misspecification in the neighborhood of the reference IS curve.

\subsection{The robust interest rate rule}

The first-order conditions can be used to derive the worst case solution for output, inflation, and the interest rate. Insert the first-order conditions in the distorted Phillips curve to obtain

$$
\pi_{t}\left(1+\frac{1}{\theta}-\frac{\kappa^{2}(\sigma+\eta)(\sigma(1-\psi)+\eta)}{\lambda}\right)=e_{t}+\kappa \psi i_{t}
$$

The distorted IS curve further implies that the interest rate can be written as

$$
\begin{aligned}
i_{t} & =-\sigma\left(x_{t}-v_{t}^{x}-u_{t}\right) \\
& =\frac{\sigma \kappa}{\lambda}(\sigma(1-\psi)+\eta) \pi_{t}+\frac{\sigma^{2} \kappa \psi}{\theta}+\sigma u_{t}
\end{aligned}
$$

Substituting the interest rate into (17) gives the worst case solution for the inflation rate

$$
\pi_{t}^{\text {worst }}=\Delta_{e} e_{t}+\Delta_{u} u_{t}
$$

with

$$
\begin{aligned}
\Delta_{e} & \equiv \frac{\lambda \theta}{\lambda\left(\theta-1-\sigma^{2} \kappa^{2} \psi^{2}\right)+\theta \kappa^{2}(\sigma(1-\psi)+\eta)^{2}} \\
\Delta_{u} & \equiv \frac{\psi \lambda \theta \sigma \kappa}{\lambda\left(\theta-1-\sigma^{2} \kappa^{2} \psi^{2}\right)+\theta \kappa^{2}(\sigma(1-\psi)+\eta)^{2}}
\end{aligned}
$$

If uncertainty becomes larger ( $\theta$ falls), the central bank fears inflation in the worst case to be higher following both types of shocks. Moreover, the effect of 
uncertainty on the strength of interest rate setting becomes larger as the cost channel coefficient rises

$$
\frac{\partial \Delta_{e}}{\partial \theta}<0, \quad \frac{\partial \Delta_{u}}{\partial \theta}<0, \quad \frac{\partial\left(\left|\frac{\partial \Delta_{e}}{\partial \theta}\right|\right)}{\partial \psi}>0, \quad \frac{\partial\left(\left|\frac{\partial \Delta_{u}}{\partial \theta}\right|\right)}{\partial \psi}>0
$$

Hence, if the cost channel becomes larger, $\partial \Delta_{e} / \partial \theta$ and $\partial \Delta_{u} / \partial \theta$ increase. The worst case output dynamics are obtained by inserting this expression into the first-order condition (14)

$$
\begin{aligned}
x_{t}^{w o r s t} & =-\frac{\kappa(\sigma(1-\psi)+\eta)}{\lambda} \pi_{t}^{\text {worst }} \\
& =-\frac{\kappa(\sigma(1-\psi)+\eta)}{\lambda}\left[\Delta_{e} e_{t}+\Delta_{u} u_{t}\right]
\end{aligned}
$$

This equation conveys the basic intuition behind the result of this paper. In the presence of a cost channel, i.e. if $\psi>0$,

$$
\left|-\frac{\kappa(\sigma(1-\psi)+\eta)}{\lambda}\right|<\left|-\frac{\kappa(\sigma+\eta)}{\lambda}\right|
$$

Hence, worst-case output must contract less to restore optimality if inflation rises. A central bank that fears the worst-case to happen needs to adjust interest rates to a smaller extend than in the absence of a cost channel. Note that, under plausible parameters, $\sigma(1-\psi)+\eta>0$. Monetary policy always "leans against the wind" (Clarida, Galí, and Gertler, 1999). Suppose the central bank fears that inflation in the worst-case is high after a shock. In the absence of a cost channel, the policymaker needs to adjust interest rates strongly to fight inflation and depress output in order to meet the optimality condition (14). Hence, the policymaker's interest rate response is weaker than in the absence of a cost channel.

Result 2: The strength of interest rate adjustment is the net effect of two opposing forces. If uncertainty increases, $\pi_{t}^{\text {worst }}$ also increases and, hence, interest rates must rise stronger than under certainty to combat inflation. If the cost channel is present, i.e. if $\psi>0$, worst-case optimal output contracts less after a shock. The central bank does not need to contract the economy by rising interest rates aggressively to restore optimality. 
The detailed implications of uncertainty for interest rate setting are derived in subsequent sections. Particularly, we will specify the conditions under which the attenuation-effect of the cost channel dominates the activism-effect of uncertainty.

Substitute $\pi_{t}^{\text {worst }}$ in the $i_{t}$ equation (18) to obtain the optimal interest rate rule in the worst-case scenario

$$
i_{t}=\sigma\left(\Omega_{e} e_{t}+\Omega_{u} u_{t}\right)
$$

with the coefficients given by

$$
\begin{aligned}
\Omega_{e} & \equiv \frac{\lambda \sigma \kappa \psi+\theta \kappa(\sigma(1-\psi)+\eta)}{\lambda\left(\theta-1-\sigma^{2} \kappa^{2} \psi^{2}\right)+\theta \kappa^{2}(\sigma(1-\psi)+\eta)^{2}} \\
\Omega_{u} & \equiv \frac{\lambda(\theta-1)+\theta \kappa^{2}(\sigma(1-\psi)+\eta)^{2}+\theta \sigma \kappa^{2} \psi(\sigma(1-\psi)+\eta)}{\lambda\left(\theta-1-\sigma^{2} \kappa^{2} \psi^{2}\right)+\theta \kappa^{2}(\sigma(1-\psi)+\eta)^{2}}
\end{aligned}
$$

If we set $\psi=0$, we are back in the Leitemo and Söderström (2005) solution

$$
i_{t}=\frac{\sigma \kappa(\sigma+\eta)}{\lambda(1-1 / \theta)+\kappa^{2}(\sigma+\eta)^{2}} e_{t}+\sigma u_{t}
$$

where higher uncertainty leads the central bank to respond stronger to supply shocks but has no impact on the response to demand shocks.

\subsection{The approximating model}

If the central bank sets interest rates according to (21), monetary policy is shielded against the worst model perturbations. Note, however, that this worst case scenario is only a metaphor for the central bank's uncertainty about its reference model. If the reference model is in fact undistorted $\left(v_{t}^{\pi}=v_{t}^{x}=0\right)$ and the central bank nevertheless pursues its robust optimal policy, the outcome is referred to as the approximating model.

Insert the worst-case interest rate rule (21) into the undistorted model to obtain the solution for output and inflation in the approximating model

$$
x_{t}^{a p p r}=-\sigma^{-1} i_{t}+u_{t}=\left(1-\Omega_{u}\right) u_{t}-\Omega_{e} e_{t}
$$


and

$$
\pi_{t}^{a p p r}=\left[\kappa(\sigma+\eta)\left(1-\Omega_{u}\right)+\kappa \psi \sigma \Omega_{u}\right] u_{t}+\left[1+\sigma \kappa \psi \Omega_{e}-\kappa(\sigma+\eta) \Omega_{e}\right] e_{t}
$$

Again, if we set $\psi=0$, the solution collapses to Leitemo and Söderström's (2005) result

$$
\pi_{t}^{a p p r}=\left(1-\kappa(\sigma+\eta) \Omega_{e}\right) e_{t}=\frac{\lambda(1-1 / \theta)}{\lambda(1-1 / \theta)+\kappa^{2}(\sigma+\eta)^{2}} e_{t}
$$

The inflation rate increases in $\theta$. Hence, a higher aversion to model uncertainty makes inflation less volatile.

\section{Caution, activism, or inactiveness?}

Under uncertainty, the central bank fears that after a shock inflation is higher due to the presence of the evil agent's model distortions, see the misspecified Phillips curve (5). Optimality requires, see the trade-off (14), that output must fall to restore equilibrium. Hence, the central bank raises the interest rate to contract the economy. This interest rate adjustment is stronger than under certainty because the central bank takes the inflationary impact of the evil agent's distortions into account.

What does the existence of a cost channel of monetary transmission imply for optimal monetary policy under uncertainty? It has long been argued that uncertainty should lead to a smaller adjustment of policy rates to exogenous shocks than under certainty. Recently, this line of reasoning came under attack as the literature moved from assessing multiplicative uncertainty to general model uncertainty. Within the robust control approach, for example, higher uncertainty implies that the central bank fears that inflation is higher in the worst case scenario and, therefore, leads the central bank to adjust interest rate more vigorously than under certainty. The following sections investigate the role of the cost channel for the effect of uncertainty on interest rate setting. 


\subsection{Parameterization}

The model is calibrated to the U.S. economy for various values of the cost channel coefficient. All parameters values are presented in table (1). The discount factor $\beta$ is set to 0.99 , which is standard in the literature. Ravenna and Walsh's (2006) estimates imply a value of $\kappa=0.10$.

Table 1: Parameter values for the analytical model

\begin{tabular}{cccccccc}
\hline \hline \multicolumn{3}{c}{ model } & & & \multicolumn{2}{c}{ policy } \\
\cline { 1 - 3 } \cline { 6 - 7 } & $\kappa$ & $\sigma$ & $\eta$ & & $\lambda$ & $\theta$ \\
0.99 & 0.10 & 1.8 & 1 & & 0.25 & {$[40,1]$} \\
\hline
\end{tabular}

The real interest rate-sensitivity of aggregate demand, $\sigma^{-1}$, is crucial in this context. Lubik and Schorfheide (2004), for example, find $\sigma=1.86$ in a post1982 sample for the US economy. Recently, Levin et al. (2005) use Bayesian techniques to estimate a sticky-price model with various sources of uncertainty which, in an extension of the basic specification, also features a working capital channel of monetary transmission. They obtain an estimate of $\sigma=2.04$ for the US economy. In this paper we use $\sigma=1.8$ as the baseline specification.

With $\lambda=0.25$, we employ a both plausible and widely used value for the output weight in the central bank's objective function. Our measure of model uncertainty, $\theta$, is varied between 40 and 1 .

\subsection{Uncertainty and interest rate setting}

How does the degree of uncertainty affect the strength of interest rate adjustment? As discussed earlier, the cost channel, in general, dampens interest rate movements. We will now analyze under which conditions the presence of a cost channel of monetary transmission affects the debate about attenuation versus anti-attenuation of policy. The interest rate response to cost-push shocks reacts to the degree of uncertainty as described by the derivative

$$
\frac{\partial \Omega_{e}}{\partial \theta}=\left[(\sigma(1-\psi)+\eta)\left(-1-\sigma^{2} \kappa \psi^{2}\right)-\lambda \sigma \psi-\sigma \kappa^{2} \psi(\sigma(1-\psi)+\eta)\right] \Pi
$$


and the interest rate response to demand shocks is affected by the degree of uncertainty as given by

$$
\begin{aligned}
\frac{\partial \Omega_{u}}{\partial \theta} & =\psi\left[(\sigma(1-\psi)+\eta)\left(-1-\sigma^{2} \kappa \psi^{2}\right)-\lambda \sigma \psi-\sigma \kappa^{2} \psi(\sigma(1-\psi)+\eta)\right] \Pi \\
& =\psi \frac{\partial \Omega_{e}}{\partial \theta}
\end{aligned}
$$

where $\Pi=\left[\lambda\left(\theta-1-\sigma^{2} \kappa^{2} \psi^{2}\right)+\theta \kappa^{2}(\sigma(1-\psi)+\eta)^{2}\right]^{-2}>0$. We restrict the analysis to positive values of $\psi$. It follows that $\partial \Omega_{e} / \partial \theta=0$ and $\partial \Omega_{u} / \partial \theta=0$ have one common solution $\bar{\psi}$, while $\psi=0$ additionally solves $\partial \Omega_{u} / \partial \theta=0$. Figure (1) plots the derivatives $\partial \Omega_{e} / \partial \theta$ and $\partial \Omega_{u} / \partial \theta$ for plausible realizations of the cost channel coefficient.

Consider first the response to cost-push shocks and its sensitivity to the degree of model uncertainty. Below the threshold $\bar{\psi}, \partial \Omega_{e} / \partial \theta<0$. Hence, interest rates are raised stronger if uncertainty increases. Beyond the threshold, the adjustment is dampened if uncertainty increases, $\partial \Omega_{e} / \partial \theta>0$. Note, however, that in this range the interest rate is lowered to combat an inflationary cost-push shock. Hence, a positive derivative with respect to $\theta$ means that larger uncertainty $(\theta$ falls) leads interest rates to be reduced more strongly. Figure (2) visualizes that, in general, higher uncertainty leads to more pronounced interest rate response to supply shocks.

Result 3: The interest rate response to a supply shock becomes more aggressive if uncertainty increases. If the cost channel is less important, the interest rate rises stronger if the central bank becomes more uncertain. If the cost channel is very important for inflation dynamics, larger model uncertainty leads to a stronger interest rate reduction than under certainty.

Hence, only the sign of the response switches, the policy reaction becomes more aggressive in either case. As becomes apparent in subsequent sections, the negative interest rate response to inflationary shocks is due to the simplistic model structure and disappears in the dynamic model to be presented below. 
The reaction to the $u_{t}$ shock is affected in the following way. A central bank which pursues a robust policy rule adjusts interest rates less aggressively to a demand shock if the cost channel exceeds the threshold $\bar{\psi}$. Hence, in this range the seminal Brainard principle holds.

The intuition behind this finding is the following. Under model uncertainty the central bank fears inflation to be higher following a demand shock than under certainty about the underlying model structure. Hence, the policymaker wants to adjust the interest rate stronger. With a cost channel present, however, strong interest rate adjustments are penalized in terms of additional inflationary pressure. It was already shown that worst-case optimal output improves after a shock. Hence, the central bank does not need to contract the economy by rising interest rates aggressively to restore optimality.

Result 4: The interest rate response to a demand shock is humpshaped in the cost channel coefficient. An empirically plausible cost channel coefficient implies that a more robust policy leads to a weaker interest rate response and secures that the legendary Brainard result carries over to this context.

Figure (3) displays the interest rate response to a positive demand shock. If the cost channel is absent, i.e. if $\psi=0$, the response equals $\sigma$ as in the model of Leitemo and Söderström (2005). In this case, uncertainty plays no role for the stance of monetary policy facing a demand shock. Policy neutralizes the effect of demand shocks and adjusts interest rates by $\sigma$ such that the net effect on the IS curve is zero.

Note that a $\psi$ coefficient slightly below two suffices to corroborate the Brainard result for the reaction to demand shocks. In this case $\partial \Omega_{u} / \partial \theta>0$, i.e. more uncertainty reduces the strength of the interest rate response to shocks.

Ravenna and Walsh (2006) estimate an augmented Phillips curve with U.S. data and obtain an estimate for $\psi$ of 1.276. As such, the estimate lies below the critical threshold $\bar{\psi}$. These estimates are, however, surrounded by considerable uncertainty. An interval of two standard errors around their estimate spans a 
range of plausible cost channel coefficients of

$$
\psi \epsilon[0.2770,2.2610]
$$

The threshold $\bar{\psi}$ falls within this range. With an alternative set of instruments, these authors obtain a considerably larger estimate for $\psi$ of 1.915. Therefore, the findings in this paper pertain to a realistic scenario facing policymakers. In addition, the dynamic model analyzed below shows that, if shocks exhibit a reasonable amount of persistence, the attenuation effect prevails already at Ravenna and Walsh's estimate of $\psi=1.276$.

\subsection{Uncertainty and welfare}

Let's now consider the variances of output, inflation, and interest rates. The interest rate variance is depicted in figure (4). We see that a higher degree of robustness increases interest rate variance. We also clearly see, as suggested before, the range in which uncertainty has no impact on interest rate volatility because the presence of a cost channel neutralizes the more active policy needed under robustness.

The variance of inflation, see figure (5), is falling in the degree of uncertainty and hump-shaped in the importance of the cost channel . Around the threshold value of $\psi$, monetary policy does not react and hence an expansionary cost-push shock feeds into inflation without being dampened. Hence, around this region the variance is unity and corresponds to those of the shock process. As the cost channel becomes less important, interest rates are used to fight cost-shocks and the economy is stabilized. As a result, inflation volatility increases if $\psi$ lies below the threshold value. If the cost channel is very important and $\psi$ lies above its threshold $\hat{\psi}$, the central bank also uses the interest rate to stabilize the economy. Hence, inflation volatility falls. An intermediate impact of interest rate on firms' marginal cost leads to a higher inflation response than a small or a very large impact. Why is this the case? The response of the inflation rate to a cost shock becomes smaller if the central bank's desire for robustness increases. Since the 
central bank adjusts interest rates stronger if uncertainty becomes larger, shocks are stabilized more and inflation volatility decreases.

The variance of the output gap in the approximating model is visualized in figure (6) and closely corresponds to the pattern of interest rate volatility. Without the cost channel, output variance drastically increases if the central bank's desire for robustness grows, i.e. if $\theta$ falls. With a high but nevertheless plausible cost channel coefficient, however, the effect of uncertainty on output gap variance is muted.

\section{Robust policy in a dynamic model}

The model considered thusfar illustrates the basic principles. It was solved under some simplifying assumptions. Among them was the restriction that the evil agent and the policymaker are restricted to respond to the same set of variables, i.e. the two kind of shocks. In this section we relax this simplifying and non innocuous assumption and allow the evil agent to respond also to lagged state variables and introduce persistence into the shock processes.

The processes driving the cost-push shock $e_{t}$ and the demand shock $u_{t}$ are now given by

$$
\begin{aligned}
& e_{t}=\rho_{e} e_{t-1}+\Sigma_{e} \varepsilon_{t}^{e} \quad \text { with } 0 \leq \rho_{e}<1, \varepsilon_{t}^{e} \sim \text { i.i.d. }(0,1) \\
& u_{t}=\rho_{u} u_{t-1}+\Sigma_{u} \varepsilon_{t}^{u} \quad \text { with } 0 \leq \rho_{u}<1, \varepsilon_{t}^{u} \sim \text { i.i.d. }(0,1)
\end{aligned}
$$

The reference model can be compactly written in state space form. The complete optimization problem thus becomes

$$
\begin{array}{cc} 
& \min _{\{i\}_{0}^{\infty}} \max _{\{v\}_{1}^{\infty}} E_{0} \sum_{t=0}^{\infty} \beta^{t}\left(\pi_{t}^{2}+\lambda y_{t}^{2}\right) \\
\text { s.t. } & \mathbf{x}_{t+1}= \\
& \mathbf{A} \mathbf{x}_{t}+\mathbf{B} i_{t}+\mathbf{C}\left(\varepsilon_{t+1}+\mathbf{v}_{t+1}\right) \\
& E_{0} \sum_{t=0}^{\infty} \beta^{t} \mathbf{v}_{t}^{\prime} \mathbf{v}_{t} \leq \eta
\end{array}
$$

The vector $\mathbf{x}_{t}=\left[\mathbf{x}_{1 t}^{\prime}, \mathbf{x}_{2 t}^{\prime}\right]^{\prime}$ summarizes both the predetermined and the forwardlooking variables and $\mathbf{A}, \mathbf{B}$, and $\mathbf{C}$ are appropriately defined matrices. The $2 \times 1$ 
vector $\mathbf{x}_{1 t}$ collects the predetermined variables $u_{t}$ and $e_{t}$ with $\mathbf{x}_{10}$ given, and $\mathbf{x}_{2 t}$ is a $2 \times 1$ vector containing the forward-looking variables $x_{t}$ and $\pi_{t}$. Finally, the $2 \times 1$ vector $\varepsilon_{t}$ contains the white-noise innovations $\varepsilon_{t}^{u}$ and $\varepsilon_{t}^{e}$. The constraint can be inserted to obtain the Lagrangian

$$
\begin{aligned}
& \min _{\{i\}_{0}^{\infty}} \max _{\{v\}_{1}^{\infty}} E_{0} \sum_{t=0}^{\infty} \beta^{t}\left[\pi_{t}^{2}+\lambda_{y} y_{t}^{2}-\theta\left(\mathbf{v}_{t}^{\prime} \mathbf{v}_{t}\right)\right] \\
& \text { s.t. } \mathbf{x}_{t+1}=\mathbf{A} \mathbf{x}_{t}+\mathbf{B} i_{t}+\mathbf{C}\left(\varepsilon_{t+1}+\mathbf{v}_{t+1}\right)
\end{aligned}
$$

The evil agent's $(2 \times 1)$ control vector is allowed to feed back on the history of the economy's state variables $\mathbf{x}_{t}$

$$
\mathbf{v}_{t+1}=f_{t}\left(\mathbf{x}_{t}, \mathbf{x}_{t-1}, \ldots\right)
$$

where $f_{t}$ is a sequence of functions. In fact, misspecifications can distort the model parameters, the autocorrelation properties of the error terms, and can introduce non-linearities. The model can be solved using Giordani and Söderlind's (2004) algorithms.

Table (2) reports the parameters used in the calibration. Apart from the persistence properties of the shock processes, the numbers are identical to those used in the previous sections. In addition, the cost channel is reflected in $\psi=1.276$

\begin{tabular}{|c|c|c|c|c|c|c|c|c|}
\hline \multicolumn{5}{|c|}{ model } & \multicolumn{2}{|c|}{ shocks } & \multicolumn{2}{|c|}{ policy } \\
\hline$\beta$ & $\kappa$ & $\sigma$ & $\eta$ & $\psi$ & $\Sigma_{e}=\Sigma_{u}$ & $\rho_{e}=\rho_{u}$ & $\lambda$ & $\theta$ \\
\hline 0.99 & 0.10 & 1.8 & 1 & 1.276 & 1.00 & 0.40 & 0.25 & 15.2 \\
\hline
\end{tabular}
as estimated by Ravenna and Walsh (2006).

Table 2: Parameter values for the model simulation

Figure (8) shows the impulse response functions for the interest rate after a cost-push shock and a demand shock. Model uncertainty induces a substantially more aggressive interest rate response than under the rational expectations case. However, without a cost channel the impact of uncertainty aversion and, hence, the difference between the robust interest rate rule and the rational expectations case is much more pronounced than under the scenario with the cost channel. 
This parameter $\theta$ is bounded only by 0 with rational expectations corresponding to $\theta \rightarrow \infty$. Hence, we have no reasonable a priori range over which we should perfom the simulation. To overcome the problem of specifying a range for $\theta$, we follow Hansen and Sargent (2005, chapter 8) and employ what they refer to as a detection error probability approach. Zero robustness, i.e. the rational expectations case, corresponds to a detection error probability of 0.5. We calculate this probability and invert it to obtain a context-specific value of $\theta$. It turns out that specifying $\theta=15.2$ corresponds to a detection error probability of around 0.20 , see figure (7), and implies only a small departure from rational expectations. Hence, the policymaker underlying this simulation is not concerned about absurdly pessimistic scenarios but considers slight deviations from the rational expectations benchmark.

The impulse responses to a cost-push shock are presented in the first two rows of figure (8). In a situation without a cost channel, the interest rate adjustment under robust policy is stronger than under rational expectations. If a cost channel is present, the interest rate adjustment is smaller since interest rate movements are costly in terms of inflationary pressure. In this situation, interest rates react stronger to a supply shock under certainty than under robust policy. As a result, inflation is stabilized less than in the absence of a cost channel. Most importantly, this result is obtained under an empirically supported value of the cost channel of 1.276, which lies far below the value that was necessary to obtain the attenuation result in the analytical model of the first part of the paper.

The impulse responses after a demand shock also corroborate the analytical findings derived in the previous sections. In the absence of a cost channel, uncertainty has no effect on the response to a demand shock since the robust response and the rational expectations response are identical. If there is no cost channel, the central bank always stabilizes demand shock by adjusting $\partial i_{t} / \partial u_{t}=\sigma$ as derived in the analytical model. For this reason, $\partial i_{t} / \partial \theta=0$. With a cost channel, however, the Brainard result is restored. The third row in figure (8) illustrates that model uncertainty leads the central bank to adjust its policy instrument less than under certainty. Moreover, in the presence of a cost channel, the response 
of inflation and output is dampened when compared to the rational expectations case.

Hence, model uncertainty motivates a cautions monetary policy stance. We can therefore state an additional finding.

Result 5: A robust policy rule derived from a rich dynamic model supports the basic findings. Model uncertainty can induce an attenuated policy response to shocks and, hence, can restore the seminal Brainard result.

Consistently with results derived above, the presence of a cost channel of monetary transmission reduces the impact of model uncertainty on interest rate setting behavior and can even restore the case for cautious monetary policy under uncertainty as suggested by Brainard (1967).

\section{Conclusions}

This paper derived robustly optimal monetary policy for an economy, in which a cost channel provides an additional channel of monetary transmission. Two forces determine the strength of interest rate adjustment. On the one hand, model uncertainty generally leads the central bank to adjust its policy instrument more aggressively than under certainty. On the other hand, the presence of a cost channel generally dampens interest rate responses to shocks. In this paper, we analyzed the net effect of these two forces and characterized the resulting interest rate setting behavior. The model is simple enough to facilitate an analytical closed form solution. We find that under plausible parameter values, the cost channel can offset the activist policy stance. In this case, uncertainty does not matter for optimal policy or can even lead to an attenuated policy stance. Under a high but still plausible realization of the cost channel coefficient, the central bank's response to demand shocks is muted as the degree of robustness increases. Hence, the paper provides a simple example in which the seminal findings of Brainard (1967) apply to robust monetary policy under model uncertainty. This 
findings supports the claim made by Blinder (1998, p. 12): "My intuition tells me that [the Brainard principle] is more general -or at least more wise- in the real world than the mathematics will support. And I certainly hope it is, for I can tell you that I was never far from my mind when I occupied the Vice Chairman's office at the Federal Reserve".

In the present framework, monetary policy operates under discretion taking private sector expectations as given. An interesting question relates to the gains from monetary commitment in the presence of model uncertainty. How should the central banker guide expectations formation of price setters and consumers if he does not fully trust his reference model? How is optimal stabilization policy affected if the private sector is uncertain about the underlying model and forms expectations based on a potentially misspecified model? Dennis (2006, 2007) elaborates on these questions und finds that the anti-attenuation result continues to hold. Hence, the presence of a cost channel could, in principle, important implications for commitment policy under model uncertainty. Future research is needed to assess whether the results provided in this paper carry over to monetary policy under commitment.

\section{References}

[1] Barth, M. J. and V. A. Ramey (2001): "The cost channel of monetary transmission", NBER Macroeconomics Annual 16, 199-240.

[2] Blinder, A. S. (1997): "What Central Bankers Could Learn from Academics - and Vice Versa", Journal of Economic Perspectives 11, 3-19.

[3] Blinder, A. (1998): Central Banking in Theory and Practice, MIT Press: Cambridge.

[4] Brainard, W. (1967): "Uncertainty and the Effectiveness of Policy", American Economic Review 57, 411-425. 
[5] Chowdhury, I., M. Hoffmann, and A. Schabert (2006): "Inflation Dynamics and the Cost Channel of Monetary Transmission", European Economic Review 50, 995-1016.

[6] Christiano, L., M. Eichenbaum, and C. L. Evans (2005): "Nominal Rigidities and the Dynamic Effects of a Shock to Monetary Policy", Journal of Political Economy 113, 1-45.

[7] Clarida, R., J. Galí, and M. Gertler (1999): "The Science of Monetary Policy: A New Keynesian Perspective", Journal of Economic Literature 37, 1661-1707.

[8] Dennis, R. (2006): "Robust Control with Commitment: A Modification to Hansen-Sargent", Working Paper, No. 2005-20, Federal Reserve Bank of San Francisco.

[9] Dennis, R. (2007): "Model Uncertainty and Monetary Policy", Working Paper, No. 2007-09, Federal Reserve Bank of San Francisco.

[10] Giannoni, M. P. (2002): "Does Model Uncertainty Justify Caution? Robust Optimal Monetary Policy in a Forward-Looking Model", Macroeconomic Dynamics 6, 111-144.

[11] Giannoni, M. P. (2005): "Robust Optimal Monetary Policy in a ForwardLooking Model with Parameter and Shock Uncertainty", forthcoming, Journal of Applied Econometrics.

[12] Giordani, P. and P. Söderlind (2004): "Solution of macromodels with Hansen-Sargent robust policies: some extensions", Journal of Economic Dynamics and Control 28, 2367-2397.

[13] Hansen, L. P. and T. J. Sargent (2005): "Robustness", book manuscript, available at http://homepages.nyu.edu/ ${ }^{\sim}$ s $43 /$.

[14] Leitemo, K. and U. Söderström (2005): "Robust Monetary Policy in the New-Keynesian Framework", forthcoming, Macroeconomic Dynamics. 
[15] Levin. A. T., A. Onatski, J. C. Williams, and N. Williams (2005): "Monetary Policy Under Uncertainty in Micro-Founded Macroeconometric Models", NBER Macroeconomics Annual 20, 229-287.

[16] Lubik, T. A. and F. Schorfheide (2004): "Testing for Indeterminacy: An Application to U.S. Monetary Policy", American Economic Review 94, 190217.

[17] Onatski, A. and J. H. Stock (2002): "Robust Monetary Policy Under Model Uncertainty in a Small Model of the U.S. Economy", Macroeconomic Dynamics 6, 85-110.

[18] Ravenna, F. and C. E. Walsh (2006): "Optimal Monetary Policy with the Cost Channel", Journal of Monetary Economics 53, 199-216.

[19] Sack, B. (2000): "Does the Fed act gradually? A VAR analysis", Journal of Monetary Economics 46, 229-256.

[20] Söderström, U. (2002): "Monetary Policy with Uncertain Parameters", Scandinavian Journal of Economics 104, 125-145.

[21] Tetlow, R. J. and P. von zur Mühlen (2001): "Robust monetary policy with misspecified models: Does model uncertainty always call for attenuated policy?", Journal of Economic Dynamics and Control 25, 911-949.

[22] Walsh, C. E. (2004): "Implications of a Changing Economic Structure for the Strategy of Monetary Policy", paper presented at the Federal Reserve Bank of Kansas City's 2003 Jackson Hole Conference.

[23] Wieland, V. (2000): "Monetary policy, parameter uncertainty and optimal learning", Journal of Monetary Economics 46, 199-228.

[24] Woodford, M. (2003): Interest and Prices, Princeton University Press: Princeton. 
[25] Žaković, S., V. Wieland, and B. Rustem (2005): "Stochastic Optimization and Worst-Case Analysis in Monetary Policy Design", forthcoming, Computational Economics. 


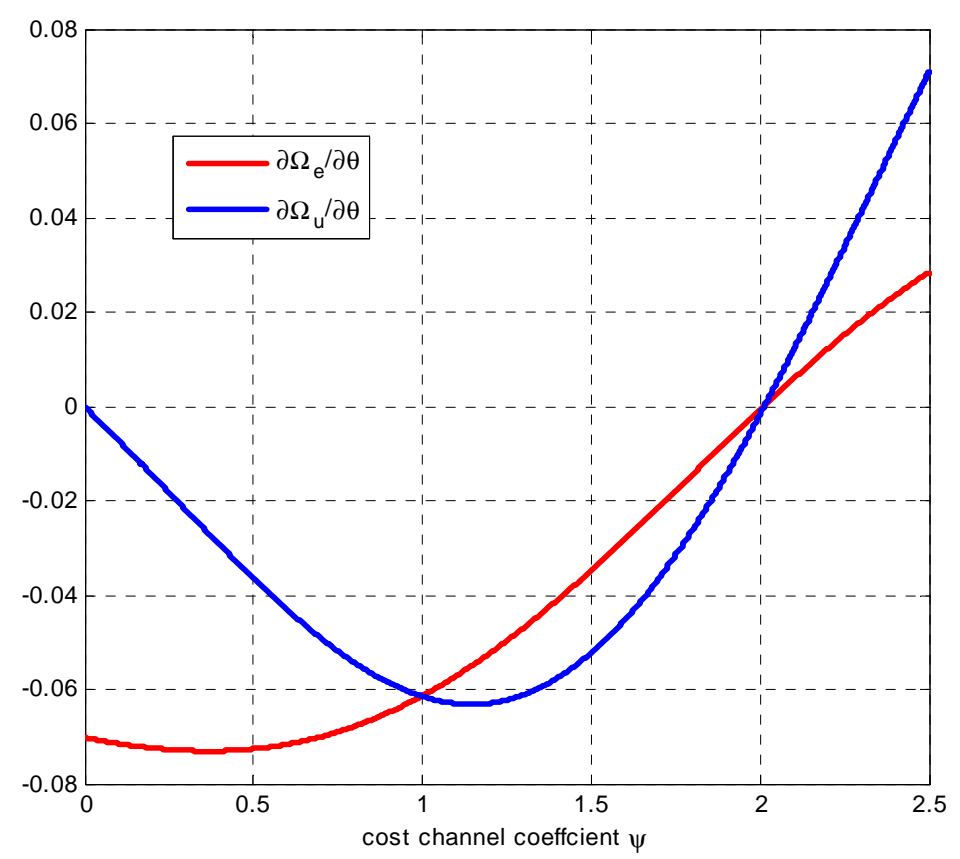

Figure 1: Derivatives of $\Omega_{e}$ and $\Omega_{u}$ with respect to the degree of model uncertainty $\theta$ for plausible realizations of the cost channel coefficient 


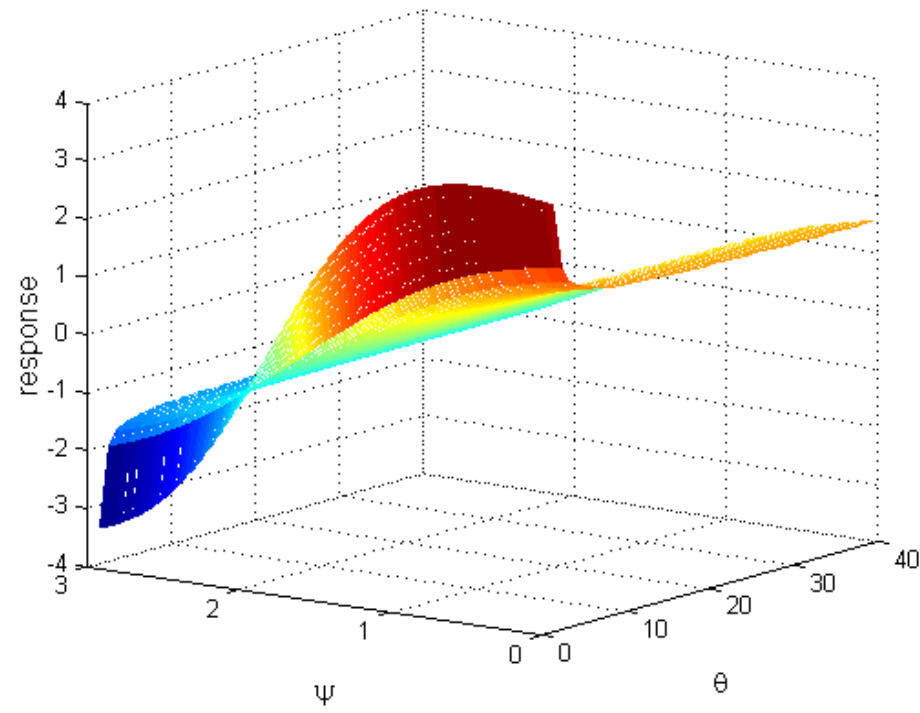

Figure 2: Interest rate response to cost-push shock

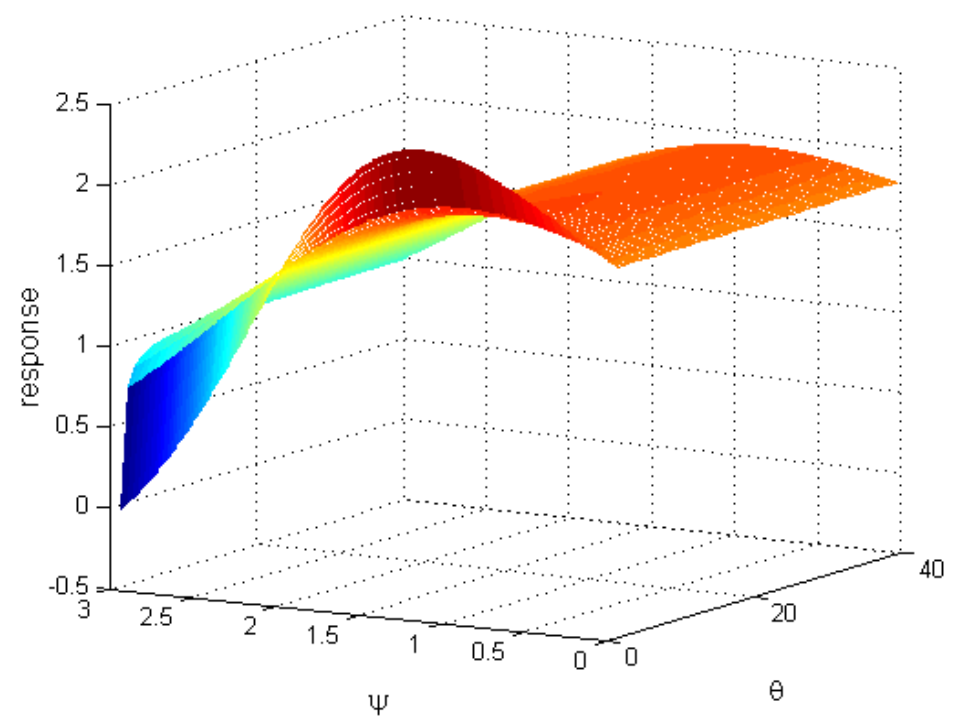

Figure 3: Interest rate response to demand shock 


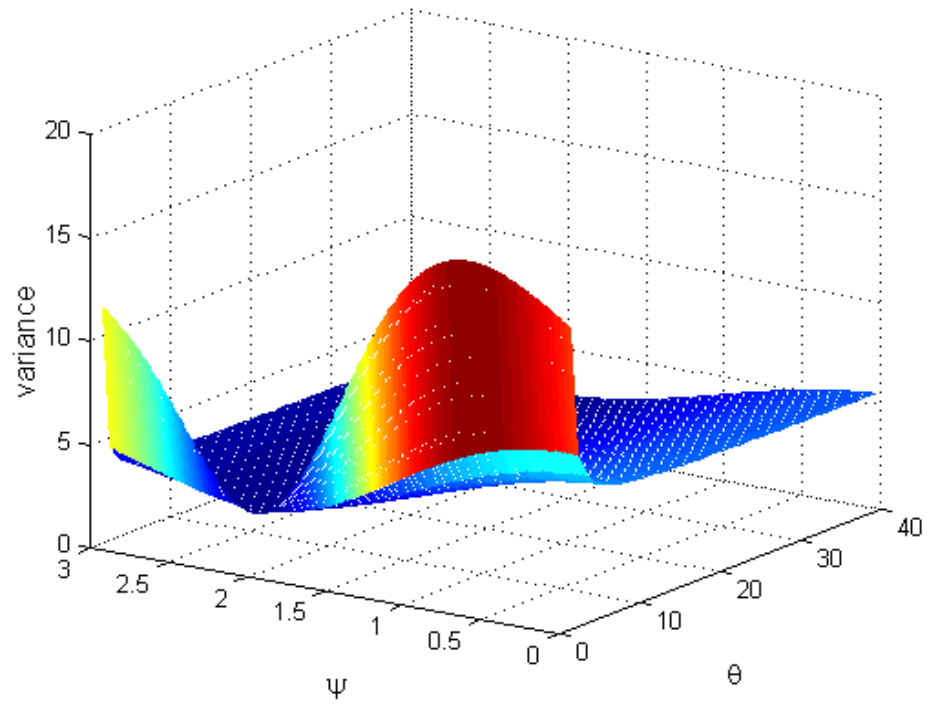

Figure 4: Interest rate variance

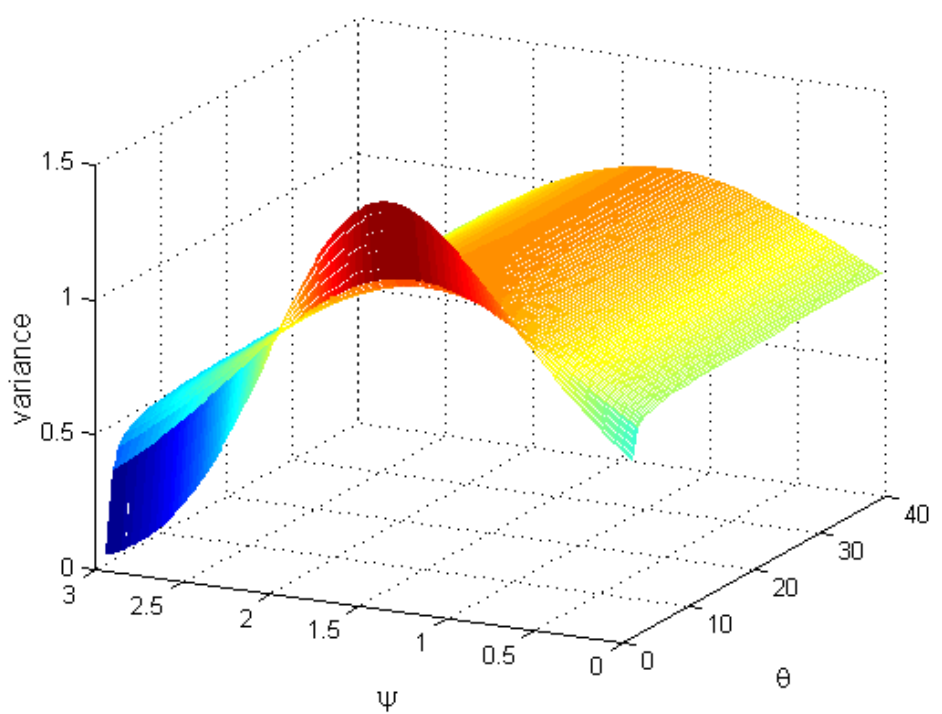

Figure 5: Inflation variance 


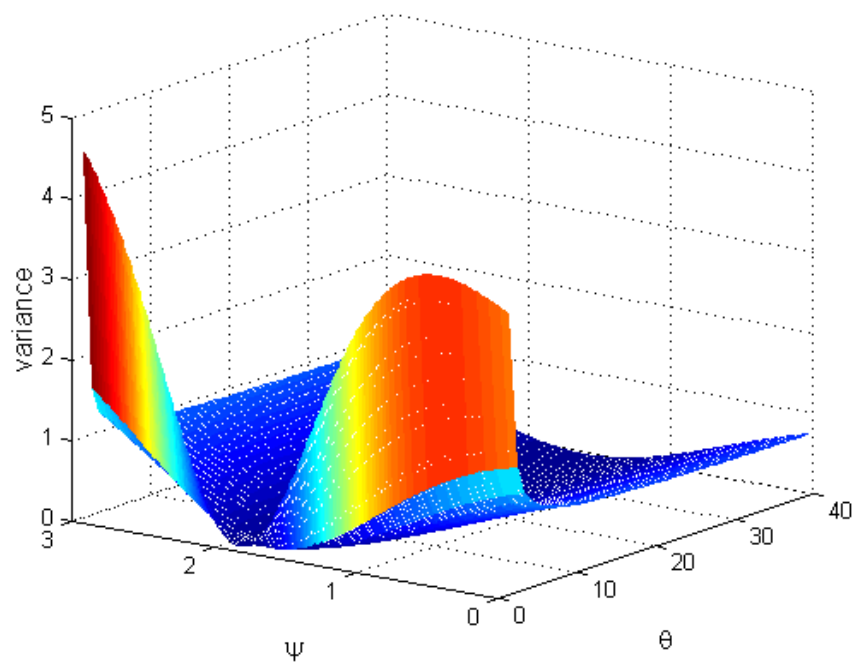

Figure 6: Output gap variance

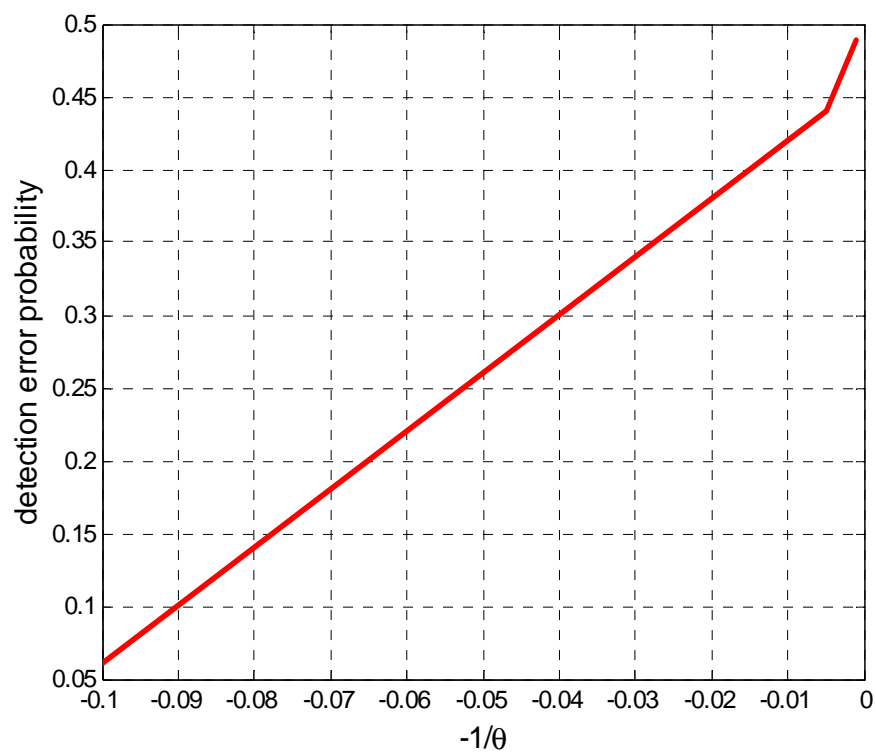

Figure 7: Detection error probability based on 10.000 simulations for a sample of 100 observations 

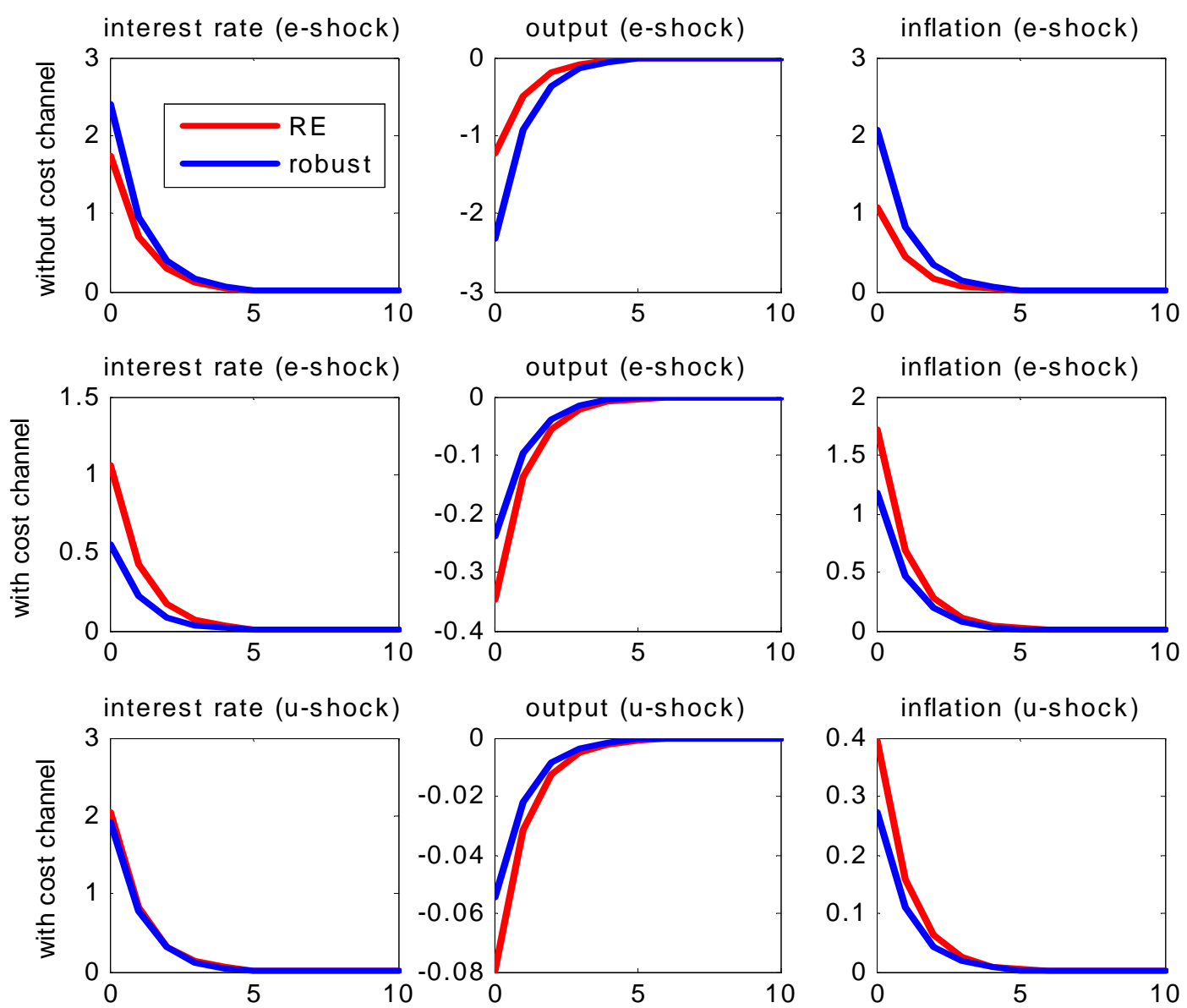

Figure 8: Impulse response functions for a cost push shock $\left(e_{t}\right)$ and a demand shock $\left(u_{t}\right)$ under rational expectations $(\mathrm{RE})$ and for robust policy under the approximating model (robust) 